\title{
Experimental analysis of gradient of negative temperature for polypropylene fiber concrete U-shaped girder
}

\author{
Xu Dong ${ }^{1,2, *}$, Fengkun $\mathrm{Cui}^{1}$, Xiudong $\mathrm{Li}^{2}$, and Songji $\mathrm{Xu}^{3}$ \\ ${ }^{1}$ School of Civil Engineering, Shandong Jiaotong University, Jinan 250357, China \\ ${ }^{2}$ China Railway 14th Bureau Group Co., Ltd, Jinan 250101, China \\ ${ }^{3}$ Shandong Hi-speed Hubei Development Co., Ltd, Wuhan 430010, China
}

\begin{abstract}
To study the negative temperature gradient models of a rail transit U-shaped girder during the winter season, a U-shaped rail transit girder was researched in Qingdao. The temperature field of the midspan section was observed for a 48 -h period during the winter. The maximum vertical and horizontal temperature difference distributions were obtained, and the negative temperature gradient models for the winter were established. The results show that the vertical temperature gradient models of the web and bottom slab should be considered. The vertical temperature gradient model of the web is a piecewise function composed of exponential and linear functions. The vertical temperature gradient model of the bottom slab is an exponential function. The transverse temperature gradient of the web is obvious, whereas the transverse temperature gradient of the bottom slab is slight.
\end{abstract}

\section{Introduction}

A pre-stressed concrete structure is significantly affected by environmental temperature changes and material heat conduction, which generate a non-uniform temperature field and temperature stress, the latter of which is considered an important reason for the cracks in a structure $[1,2]$. Meanwhile, study results have indicated that a structure has positive temperature gradients caused by a temperature increase in the environment, resulting from the surface temperature of the structure being higher than the internal temperature, and that the effects of a negative temperature gradient caused by a decrease in temperature should not be ignored $[3,4]$.

A U-shaped girder is a new type of structure used in urban rail transit viaduct bridges in China, as shown in Figure 1, and was recently derived from a foreign groove girder. Compared with many traditional bridge components, such as T-shaped and box girders, a Ushaped girder has an open thin-walled U-shaped section, which is composed of a bottom slab, web, and flange slab. Therefore, the distributions of the temperature field can be affected by the open U-shaped section and have their own particularity. A number of scholars have conducted relevant researches into the regularity of the temperature field distributions. Song et al established a thermodynamics model and proposed a sunshine temperature distribution curve of a trough-shaped section based on a cable-stayed bridge, respectively $[5,6]$. Quan investigated the temperature distributions of a U-shaped girder based on bridge monitoring, and obtained the vertical temperature gradient parameter measurements of the railway bridge design code [7]. Xu put forward the vertical and transverse temperature distributions during the summer through a field test, and established positive temperature gradient models [8].

In conclusion, although many scholars have studied the temperature field distributions of trough-shaped and U-shaped girders, studies on the temperature field of a U-shaped girder during winter remain insufficient. Although positive and negative temperature gradient models have been proposed for many different bridge design codes in various countries, they are all based on a box girder structure, and the temperature gradient models of a U-shaped girder have not been specified. Therefore, relevant researches on simple and accurate temperature gradient models of a U-shaped girder that can be used in an engineering design should be conducted.

In this paper, a polypropylene fiber concrete Ushaped girder used in the Chinese city of Qingdao was researched. The temperature field of the midspan section within a 48 -h period was observed during the winter. The maximum vertical and horizontal temperature difference distributions were obtained, and negative temperature gradient models for winter were established. All results were shown to be significant for use in an engineering design.

\section{Project Details}

This study is based on the Blue Silicon Valley intercity rail transit project in Qingdao. As shown in Figure 1, Ushaped girders using pre-stressed concrete as a simple support structure with a span length of $30 \mathrm{~m}$ were used. The open thin-walled U-shaped section was composed of 
a bottom slab, web, and flange slab. As shown in Figure 2 , at the mid-span section of the girder, the height is 1.8 $\mathrm{m}$, the width of the top is $5.32 \mathrm{~m}$, the width of the bottom is $3.98 \mathrm{~m}$, and the thicknesses of the web and bottom slab are both $0.26 \mathrm{~m}$. For the girder, polypropylene fiber concrete with a fiber dosage of $0.9 \mathrm{~kg} / \mathrm{m}^{3}$ and a standard strength grade of C55 was adopted. Pre-stressed steel with high strength and low relaxation was used in this girder, which has a diameter of $\Phi^{\mathrm{s}} 15.2$ and standard strength of $R_{y}^{b}=1860 \mathrm{MPa}$.

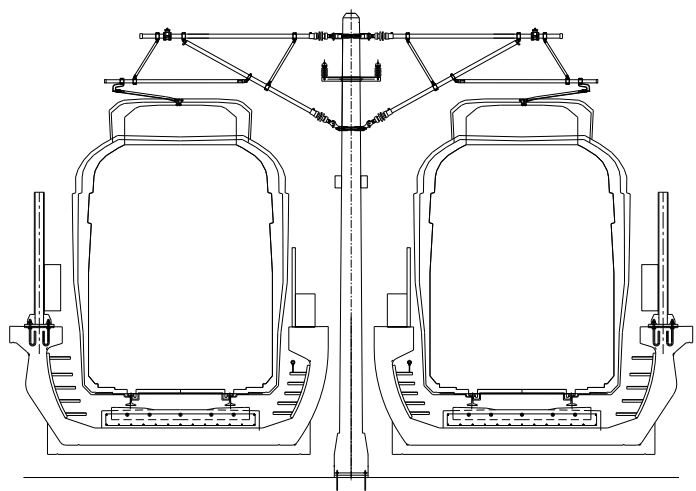

Fig. 1. Structure diagram of U-shaped girder for urban rail transit

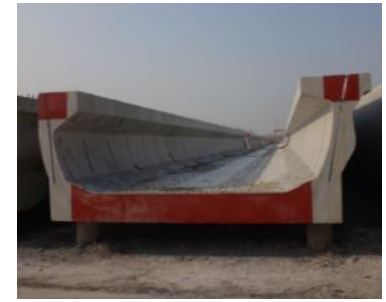

(a) U-shaped girder

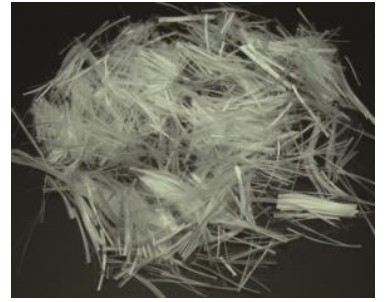

(b) Polypropylene fiber
Fig. 2. Photographs of U-shaped girder and polypropylene fiber

As shown in Figure 1 and Figure 2, a U-shaped girder differs from a traditional box girder in that it has a thin wall open structure, and the inside of the web and bottom slab are easily affected by the external environment. If temperature gradient models based on a box girder are applied to the design of a U-shaped girder, significant errors will occur in determining the actual temperature difference effect.

\section{Experiment Analysis of U-Shaped Girder Temperature Field}

\subsection{Test contents and sensor locations}

During the winter season, the temperature is lower, the solar radiation is weaker, and the negative temperature gradient of the structure caused by a decrease in temperature is obvious. To obtain regularities in the temperature field distributions and temperature difference effects, in this study, a continuous $48 \mathrm{~h}$ observation of the temperature field of a U-shaped girder was carried out in January, which is a typical winter month. The 48-h test period began at 20:00 on Jan. 13, and measurements were taken at $2 \mathrm{~h}$ intervals. The test contents included the surface and interior temperatures of the structure. The midspan section, which is the most unfavorable load section of a simple support girder, was chosen as the test section of the temperature field.

The direction of the U-shaped girder at the test site was northwest-southeast. The number of internal temperature measurement points and surface temperature measurement points were 57 and 13 respectively. In the northwest direction, the southwest was on the left side, and the northeast was on the right side. The measurement points were distributed into six groups according to their locations and function: internal temperature measurement points of the left web (LW), right web (RW), and bottom slab (BS); temperature measurement points of the left web surface (LWS), right web surface (RWS), and bottom slab surface (BSS). The locations of all measurement points are clearly shown in Figure 3 and Figure 4.

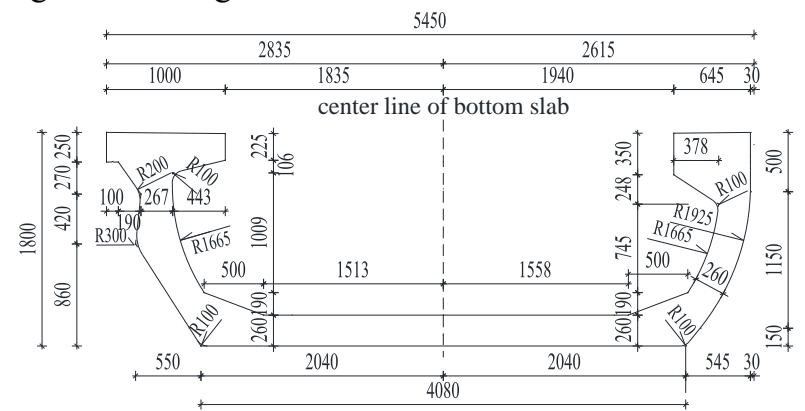

Fig. 3. Dimensions of midspan section (unit: $\mathrm{mm}$ )

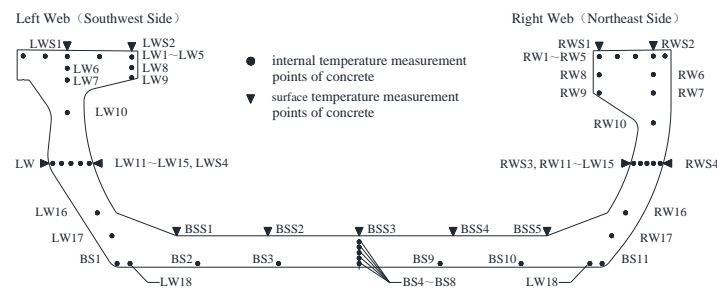

Fig. 4. Layout of measurement points

To accurately reflect the temperature distribution from the surface to the internal portion of the girder accurately, a portable infrared thermometer was used to monitor the surface temperature of the girder, embedded intelligent temperature sensors were used to monitor the internal temperature of the girder.

\subsection{Moment of maximum temperature difference}

Owing to the structural form and temperature conductivity of the material, asymmetrical temperature gradient distributions occurred concurrently in the test section of the U-shape girder, which resulted in a temperature difference. Figure 5 shows the vertical timehistory curve of the maximum temperature difference at different parts of the structure. As shown in Figure 5, the minimum temperature variations of the left and right sides of the web and the bottom slab were basically similar on both observation days. The minimum temperature of the web and bottom slab occurred at 8:00. This is not inconsistent with the time of the minimum air 
temperature, which occurred at 2:00. Based on the analysis, because the specific heat of the concrete is small, the rate of heat release is slow, and the temperature of structure becomes cold later. The minimum vertical temperature of the structural components, which occurred at 8:00 on Jan. 15, is greater than on the previous observation day. The minimum temperature of the left side of the web was $-16.5^{\circ} \mathrm{C}$, and the minimum temperature of the right side of the web was $-12.7^{\circ} \mathrm{C}$. The minimum temperature of the bottom slab was $-12{ }^{\circ} \mathrm{C}$. Because of the low heat conduction of the concrete, the internal temperature of the structure was low. Therefore, it can be concluded that the time of the maximum temperature difference in the test section was at 8:00 on the second day of observations.

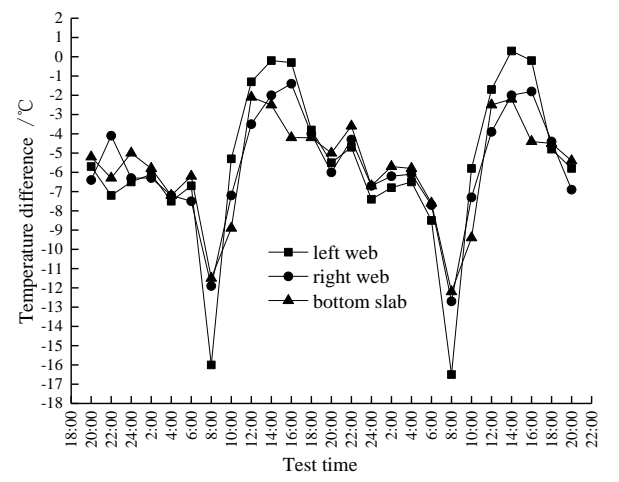

Fig. 5. Vertical time-history curve of maximum temperature difference

\subsection{Two-dimensional temperature distribution}

Many researches have shown that the effect of the temperature difference throughout the length of a bridge can be ignored because certain factors, such as the location, intensity of sunlight, and orientation, are basically unchanged. Therefore, the temperature field of the beam can be simplified as having a two-dimensional temperature distribution along the vertical and transverse directions of the bridge.

As the test results show, the temperature of the left side of the web was lower, the temperature difference was larger, and the distribution law was clear. The test data of LWS1, LW6, LW7, LW10, LW13, and LW16LW18 recorded at 8:00 were joined, and the most disadvantageous vertical temperature field distribution of the web was obtained, as shown in Figure 6.

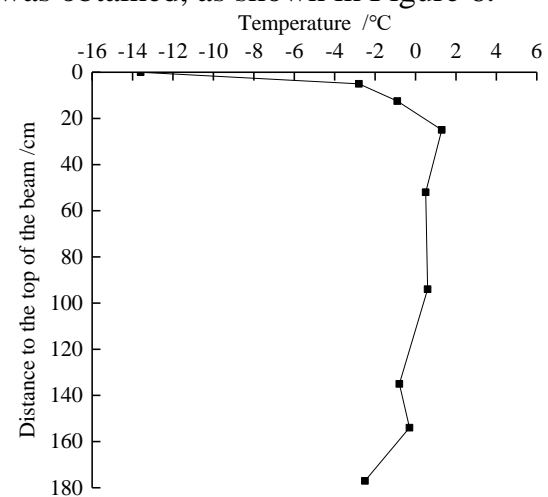

Fig. 6. Measured vertical temperature field of web
As indicated in Figure 7, the test data of BSS3 and BS4-BS8, also recorded at 8:00, were joined, and the most disadvantageous vertical temperature field distribution of the bottom slab was obtained.

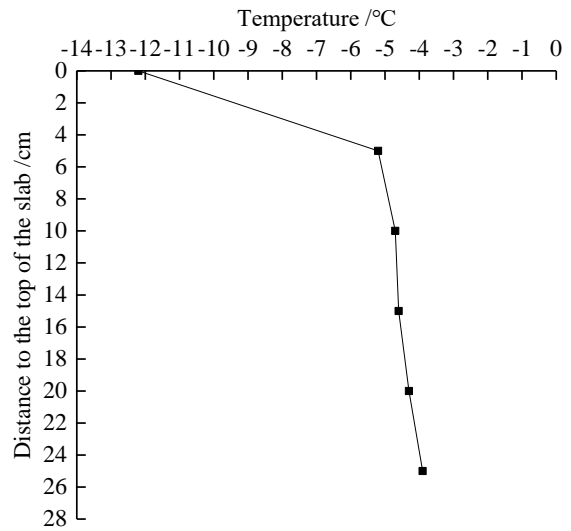

Fig. 7. Measured vertical temperature field of bottom slab

Based on the test results of the transverse temperature difference, the temperatures of the left side of the web, which were recorded at 15:00 on Jan. 15, were lower, and the temperature differences were larger. Therefore, the test data of S13, LWS3, LWS11 - LWS 15 , LWS4, and S14 recorded at 8:00 were joined, and the most disadvantageous transverse temperature field distribution of the web was obtained, which can be seen in Figure 8.

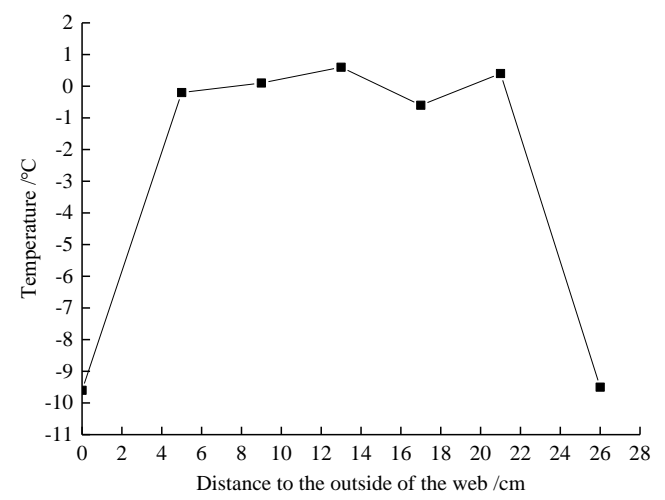

Fig. 8. Measured transverse temperature field of web

In addition, when the test data of BS1-BS3 and BS8BS11 were joined, and the most disadvantageous transverse temperature field distribution of the bottom slab was obtained, which can be seen in Figure 9.

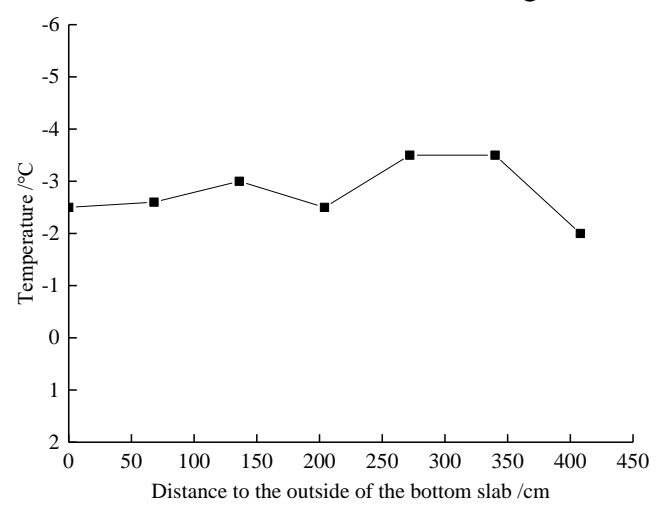

Fig. 9. Measured transverse temperature field of bottom slab 


\section{Temperature Gradient Model}

\subsection{Vertical temperature gradient}

Based on the measured vertical temperature field of the web and bottom slab, shown in Figure 6 and Figure 7, respectively, the measured vertical temperature distribution of the U-shaped girder during winter was different from the temperature gradient mode, which is an exponential function, as proposed in the railway bridge design code of China [9]. Because a U-shaped girder has an open thin-wall structure, the web and bottom slab can be affected by direct solar radiation, and the temperature difference between the two parts is greater. The web plate and bottom temperature gradient mode should be considered respectively.

Based on the measured vertical temperature field of the web, shown in Figure 6, the temperature difference of the upper web was larger, whereas that of the central part of the web was small, and that of the bottom slab was slightly lower. According to the distribution of the vertically measured temperature field and the railway bridge design code in China, the vertical temperature field of a U-shaped girder was represented using a temperature difference form. In this equation, the temperature difference of the upper web was fitted with the exponential function, and the temperature difference of the bottom web was indicated using a linear function. Therefore, the vertical temperature gradient of the web was obtained and is shown in formula (1):

$$
t_{y w}=\left\{\begin{array}{cc}
t_{m} e^{-a_{1} y} & A_{1} \leq y<B_{1} \\
\frac{t_{n}}{C_{1}-B_{1}} y-\frac{B_{1} t_{n}}{C_{1}-B_{1}} & B_{1} \leq y \leq C_{1}
\end{array}\right.
$$

where, $t_{y w}$ is the vertical temperature difference of the web $\left({ }^{\circ} \mathrm{C}\right) ; t_{m}$ is the vertical temperature difference of the upper web $\left({ }^{\circ} \mathrm{C}\right) ; t_{n}$ is the vertical temperature difference of the bottom web; $a_{1}$ is the calculation parameter of the vertical temperature gradient of the web; $A_{1}$ is the top surface coordinate of the web, which is defined as a zero coordinate; $B_{1}$ is the top surface coordinate of the bottom slab; and $C_{1}$ is the bottom surface coordinate of the bottom slab in $\mathrm{cm}$.

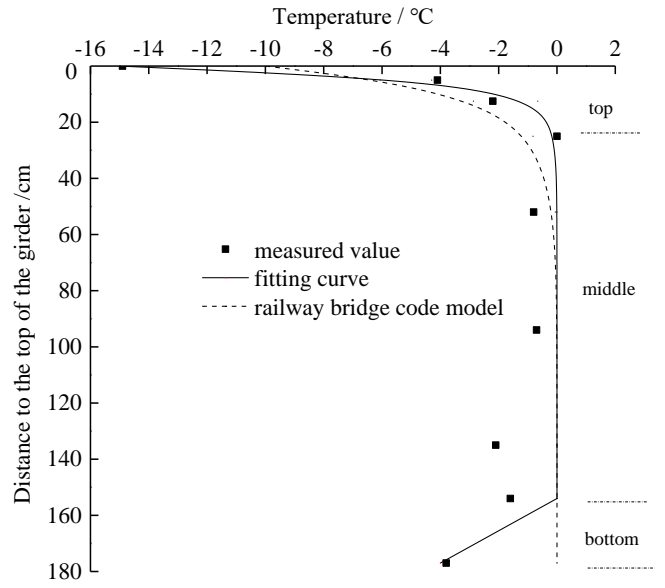

Fig. 10. Comparison of vertical temperature gradients of web
According to the structure size and test results, $t_{m}=15{ }^{\circ} \mathrm{C}, a_{1}=0.25, t_{n}=-4{ }^{\circ} \mathrm{C}, A_{1}=0 \mathrm{~cm}, B_{1}=154 \mathrm{~cm}$, and $C_{1}=180 \mathrm{~cm}$. A comparison of the different vertical temperature gradient models of the web is shown in Figure 10.

Based on the measured vertical temperature field of the bottom slab, shown in Figure 7, the upper surface temperature of the bottom slab is lower, and then rapidly increases. According to the vertical temperature field distribution of the bottom slab, the vertical temperature gradient is represented by the temperature difference. When the vertical temperature difference distribution of the bottom slab is fitted with an exponential function, the vertical temperature gradient model of the bottom slab is obtained as shown in formula (2):

$$
t_{y b}=t_{b} e^{-a_{2} y} \quad A_{2} \leq y \leq B_{2}
$$

where, $t_{y b}$ is the vertical temperature difference of the bottom slab; $t_{b}$ is the maximum temperature difference of the bottom slab $\left({ }^{\circ} \mathrm{C}\right) ; a_{2}$ is the fitting calculation parameter; $A_{2}$ is the top surface coordinate of the bottom slab, which is defined as a zero coordinate; and $B_{2}$ is the bottom surface coordinate of the bottom slab, where the direction of the coordinate is down to positive value, the unit of which is $\mathrm{cm}$.

According to the structure size and test results, $t_{b}=-8{ }^{\circ} \mathrm{C}, a_{2}=0.4, A_{2}=0 \mathrm{~cm}$, and $B_{2}=25 \mathrm{~cm}$. The vertical temperature gradient model of the bottom slab is shown in Figure 11.

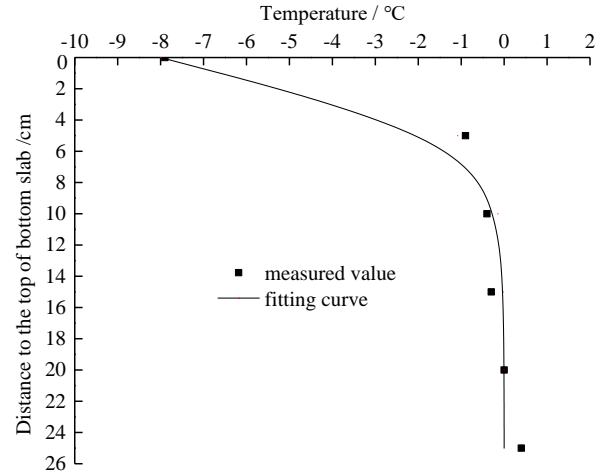

Fig. 11. Vertical temperature gradient of bottom slab

\subsection{Transverse temperature gradient}

Based on the measured transverse temperature field of the web and bottom slab, shown in Figure 8 and Figure 9, respectively, the measured temperature difference of the web of a U-shaped girder is larger during the winter, whereas the transverse temperature difference of the bottom slab is small. Therefore, only the horizontal temperature gradient of the web was considered. According to the measured transverse temperature field distribution of the right side of the web, shown in Figure 8 , the transverse temperature gradient is represented by the temperature difference. When the transverse temperature difference distribution of the web is fitted with an exponential function, the transverse temperature gradient model is obtained, as shown in formula (3): 


$$
t_{x w}=\left\{\begin{array}{cc}
t_{w} e^{-a_{3} x} & A_{3} \leq x<B_{3} \\
t_{w} e^{-a_{3}\left(C_{3}-x\right)} & B_{3} \leq x \leq C_{3}
\end{array}\right.
$$

where, $t_{x w}$ is the transverse temperature difference of the web $\left({ }^{\circ} \mathrm{C}\right) ; t_{w}$ is the maximum transverse temperature difference of the web $\left({ }^{\circ} \mathrm{C}\right) ; a_{3}$ is the fitting calculation parameter; $A_{3}$ is the outside surface coordinate of the web, which is defined as a zero coordinate; $B_{3}$ is the central coordinate of the web; and $C_{3}$ is the inside surface coordinate of the web in $\mathrm{cm}$.

According to the structure size and test results, $t_{w}=-10{ }^{\circ} \mathrm{C}, a_{3}=0.5, A_{3}=0 \mathrm{~cm}, B_{3}=17.5 \mathrm{~cm}$, and $C_{3}=25 \mathrm{~cm}$. The transverse temperature gradient model of the web is shown in Figure 12.

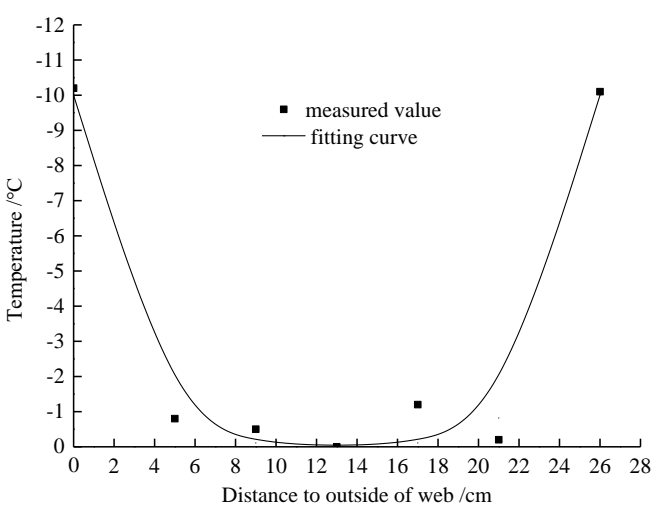

Fig. 12. Transverse temperature gradient of web

\section{Conclusion}

In this paper, a rail transit U-shaped girder was researched in Qingdao. The temperature field of a midspan section was observed for a 48-h period during the winter. The maximum vertical and horizontal temperature difference distributions were obtained, and the negative temperature gradient model for winter was established. The main conclusions can be summarized as follows:

The web and bottom slab of a U-shaped girder are influenced directly by the environment; in addition, the vertical temperature difference is larger, and the vertical temperature gradient model of the web is a piecewise function composed of exponential and linear functions. The vertical temperature gradient model of the bottom slab is an exponential function.

The measured transverse temperature gradient of the web is obvious, and the transverse temperature gradient of the bottom slab is small during winter. Therefore, only the horizontal temperature gradient of the web, which is an exponential function, is considered.

Because of the engineering confinement conditions, the temperature gradient models proposed in this paper are only applicable in northern areas of China, and can provide an important reference for the design and calculation of U-shaped girders in such areas.

\section{Acknowledgments}

The authors would like to acknowledge the financial support received from the Ministry of Transport of China (Grant No.: 2015319817280) and the support received from the Science and Technology Development Project of Shandong Province (Grant No.: 2014GGX105005).

\section{References}

1. Abid S, Taysi N, Ozakca M. Experimental analysis of temperature gradients in concrete box-girders. Construction and Building Materials, 106, 523-532 (2016)

2. Ihara I, Tomomatsu T. In-situ measurement of internal temperature distribution of sintered materials using ultrasonic technique. IOP Conference Series: Materials Science and Engineering, 18(2), 022008 (2011)

3. Gonglian D, Xuehui W, Haiting S. Study on horizontal and vertical temperature gradient of ballastless track on bridge in cold season. Journal of Huazhong University of Science \& Technology, 43(7), 1-5 (2015)

4. Bin G, Zhijian C, Xindi C. Temperature gradients in concrete box girder bridge under effect of cold wave Journal of Central South University, 21(3), 1227 1241 (2014)

5. Song X, Wenxin W. Research on vertical temperature distribution mode of U-shape beam on high-speed railway. Railway Standard Design, 58(12), 81-85 (2014)

6. Song X, Gonglian D, Bin Y. Sunshine temperature mode of prestressed concrete bridge with U-shape section. Scientia Sinica: Technologica, 03, 286-292 (2016)

7. Quan L. An experimental and theoretical research of the temperature field of rail transit concrete Ushaped beam. Nanjing Southeast University (2013)

8. Xu D, Shuchen L, Pengcheng W. Research on sun light temperature gradient effect of ballastless rail transit U-shaped beam. Journal of Harbin Engineering University, 07, 1-8 (2017)

9. MRPRC (Ministry of Railways of the People's Republic of China). TB 10002.3-2005 code for design on reinforced and prestressed concrete structure of railway bridge and culvert, Beijing China Railway Publishing House (2005) 